
Electronics and Instrumentation Engineering

(An ISO 3297: 2007 Certified Organization)

Vol. 3, Issue 8, August 2014

\title{
To Increase the Network Lifetime by Using the Relay Nodes
}

\author{
$\mathrm{Anku}^{1}$, Hardeep Singh ${ }^{2}$ \\ PG Student, Dept. of ECE, Baba Farid College of Engineering \& Technology, Deon (Bathinda), India ${ }^{1}$ \\ H.O.D, Dept. of ECE, Baba Farid College of Engineering \& Technology, Deon (Bathinda), India ${ }^{2}$
}

\begin{abstract}
A wireless sensor network is type of wireless network. Basically it consist a collection of tiny device are called sensor node, sensor node has a resource constraint means battery power, storage and communication capability. Network lifetime is the most important metric for the evaluation of sensor networks. In a resource-constrained environment, the utilization of every limited resource must be considered. The network can only fulfil its purpose as long as it is considered alive, but not after that. It is therefore an indicator for the maximum utility a sensor network can provide. Energy efficiency is therefore of paramount importance in sensor networks that are constrained by limited resources. In this paper we purposed a novel technique in which we added the no of relay nodes in the network to increase the network lifetime.
\end{abstract}

KEYWORDS: Sensor network, relay nodes, sensor nodes, lifetime, and throughput.

\section{I.INTRODUCTION}

This wireless sensor networks is depends on a simple equation: Sensing + CPU + Radio $=$ Thousands of possible applications. A wireless sensor network is type of wireless network. It is small and infrastructure less. Basically wireless sensor network consist a number of sensor node, called tiny device and these are working together to detect a region to take data about the environment. Each node in a sensor network is typically equipped with a radio transceiver or other wireless communication device, a small microcontroller, and an energy source, usually a battery. The size of a single sensor node can vary from shoebox-sized nodes down to devices the size of grain of dust. The cost of sensor nodes is similarly variable, ranging from hundreds of dollars to a few cents, depending on the size of the sensor network and the complexity required of individual sensor nodes. Size and cost constraints on sensor nodes result in corresponding constraints on resources such as energy, memory, computational speed and bandwidth, among which energy is the scarcest resource of WSN nodes. Every sensor in a WSN has a sensing range and a transmission range. An object can be discovered by a sensor if it is within the sensing range of the sensor, and two sensors can transmit data to each other if they are within each other's transmission range. Routing, one of the most energy-expensive operation, is usually multi-hop (from the source, node to node, towards the destination), due to the polynomial growth in the energy-cost of radio transmission with respect to the transmission distance.

WSNs are to be deployed in large numbers in various environments, including remote and hostile regions, with ad-hoc communications. The energy in nodes determines the lifetime of WSNs. The lifetime of WSNs will be shortened if some sensors are used more often than others as their battery power is depleted sooner. For this reason, sensor deployment, algorithms and protocols need to address lifetime maximization, robustness and fault tolerance issues. Therefore, in sensor deployment, network topology is important. To be fair, it is always better to deploy sensors in similar positions if possible. That is, every sensor has about the same number of neighbours. In this way, suppose the probability of any node becoming a source or a destination is equal, no sensor will be more frequently used as a router due to the network topology. In addition, between communicating sensors there should be multiple paths so that the network is more fault tolerant and robust. With the energy constrained nature of sensor nodes, it is very important to make efficient use of battery power in order to increase the lifetime of network [1]. In most cases, sensor nodes rely on batteries for power. Since battery replacement is very difficult if not impossible, the sensors have to operate on an extremely frugal power budget. Even in some cases where the sensors gather renewable energy from the environment, the power budget remains very similarly, other resources such as communication bandwidth and computational power are also limited. Therefore, a sensor network that is efficient in the use of resources is required. In conventional routing 


\title{
. Electronics and Instrumentation Engineering
}

\author{
(An ISO 3297: 2007 Certified Organization)
}

\section{Vol. 3, Issue 8, August 2014}

algorithms, some nodes are constantly involved in forwarding data packets, hence more energy will be depleted among those nodes and the nodes will die much earlier than others causing disconnection of the network.

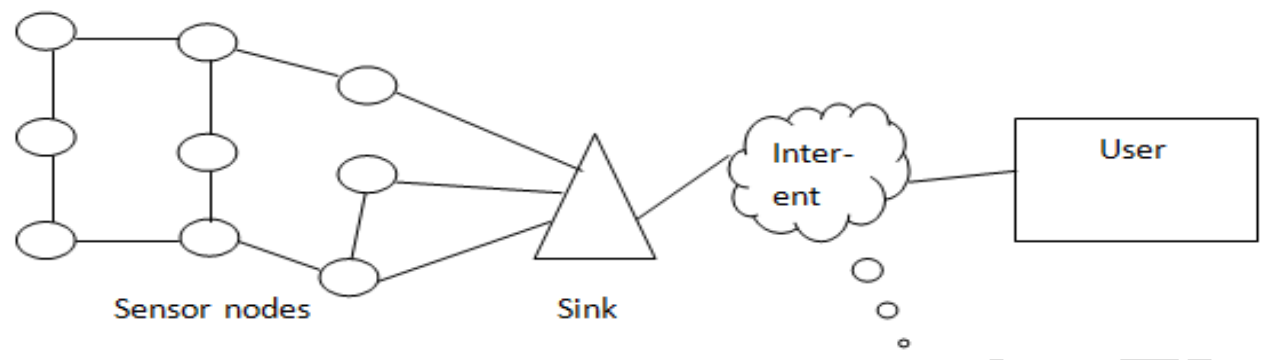

Fig. 1: Wireless sensor networks

In a WSN the node that gathers the data information refers to sink. The sink may be connected to the outside world through internet where the information can be utilized within time constraints [2]. To broadcast information to all over the network consume more nodes and more network resources. It also consumes more energy and bandwidth [4]. Sensor nodes carry limited power resources that are irreplaceable therefore; there is a need to design an energy efficient technique to increase the life of wireless sensor networks. An inbuilt trade-off mechanism should be made so that the end-user should opt for prolonging network lifetime at the cost of lower throughput or higher transmission delay [11]

\section{II.RELATED WORK}

Peyman Neamatollahi et al. [4] explained that clustering is an effective approach for organizing the network into a connected hierarchy, load balancing, and prolonging network lifetime. Clustering protocols in wireless sensor networks are classified into static and dynamic. In static clustering, clusters are formed once, forever and role of the cluster head is scheduled among the nodes in a cluster. However, in dynamic clustering the time is divided into rounds and clustering is performed in the beginning of each round. This paper presents a Hybrid Clustering Approach (HCA). Whenever a cluster head consumes a pre specified part of its energy, it indirectly informs all other nodes so, clustering will be done in the beginning of the upcoming round. Therefore, clustering is performed on demand. To evaluate the efficiency of proposal, the well known distributed clustering protocol, HEED, is used as baseline example. By means of simulation results, we demonstrate that significant energy saving can be achieved using HCA.

Jakob Salzmann et al. introduced [5] a large wireless sensor networks, low energy consumption is a major challenge. Hence, deployed nodes have to organize themselves as energy efficient as possible to avoid unnecessary sensor and transceiver operations. The energy conserving operations are limited by the task of the network; usually the network has to guarantee complete functionality during its lifetime. The contribution of this paper completes the functionality-aware and energy-efficient clustering algorithm family MASCLE by two innovative algorithms. As already given by the MASCLE algorithms, the proposed Hex-MASCLE algorithms combine advantages of temporal and spatial network fragmentation. In contrast to previous approaches, the shapes of the basic cells are given by regular hexagons, similar to honeycombs. In the present work, two possible versions for hexagon-based clustering with self-healing abilities are proposed and evaluated. As result, the applying sensor network achieve a significant improve of network lifetime. Additionally, the algorithms are more fault tolerant against localization errors.

Ewa Hansen, et al. they discussed [6] that wireless sensor networks becoming very important for developing of energy efficient infrastructure. They found the minimum separation distance between cluster heads in a cluster based sensor network, prolonging net-work lifetime by lowering the energy consumption. They performed simulation to determine how much energy is consumed by sensor network in separating the cluster heads. They also discussed the effect of energy consumption for a given minimum separation distance between cluster heads. They showed that wireless sensor network could better performed when they introducing a minimum separation distance between cluster 
 Electronics and Instrumentation Engineering}

(An ISO 3297: 2007 Certified Organization)

\section{Vol. 3, Issue 8, August 2014}

heads. It is checked by comparing the number of message was received by the base station.

K. Latif et. al [7], have presented routing technique called Divide-and-Rule which is based on static clustering and minimum distance based Cluster Head selection. Network area is logically divided into small regions (clusters). Old fashioned routing techniques such as LEACH, LEACH-C are not as energy efficient as present day clustering techniques such as Divide-and-Rule scheme. The benefit of Divide-and-Rule scheme is that when it is compared with LEACH and LEACH-C this scheme provides better results in terms of stability period, network life time, area coverage and throughput. But the limitation of this scheme is that during routing each node in Os region sends its data to Primary level Cluster Heads which then forwards the aggregated data to the secondary level Cluster Head present in the Ms. Secondary level Cluster Heads then, aggregate all collected data and forward it to Base Station which will lead to more energy consumption of $\mathrm{CH}$ nodes present in the Middle Square and Inner Square regions which may lead to energy hole and may cause data routing problems. Secondary level Cluster Heads then, aggregate all collected data and forward it to Base Station which will lead to more energy consumption of $\mathrm{CH}$ nodes present in the Middle Square and Inner Square regions which may lead to energy hole and may cause data routing problems.

Vinay Kumar [8] discussed in wireless sensor networks to maximize the lifetime of the sensor network, for the data transfer the path is selected in such a way in which the energy consumption is minimized in that path. To support high scalability and better data aggregation, sensor nodes are often grouped into clusters. Clusters create hierarchical wireless sensor network, the sensor nodes utilization their limited resources in efficient way and thus extends network lifetime. They presented taxonomy of energy efficient clustering algorithms in WSNs, and also presented timeline and description of LEACH and Its descendant in WSNs.

\section{III.SYSTEM MODEL AND ASSUMPTIONS}

A WSN composed of 50 sensor nodes. All nodes are randomly deployed into the fixed area. The first step involved in this phase is the plotting of sensor nodes in $800 * 800 \mathrm{~m}$ square region. The sensor nodes including the base station are deployed in a 2D plot. Initially, energy of about 80 joules is assigned to the each node. In existing technique, suppose we had corner node which wants to communicate with sink through intermediate nodes. First of all it sends request to its cluster head of nearest cluster. Then this cluster head further sends data to its nearest available region. The nodes which are deployed near sink are main nodes which participate to communicate with sink and intermediate nodes. The problem arises when battery of the nodes near sink goes down communication stop. Because sink node can communicate only with the help of region nearby node not directly. The main reason is that nearest sensor nodes are out of range of sink. So battery degrades and communicate stop as shown in fig.2. To overcome this problem relay nodes will be used instead of sensor nodes.

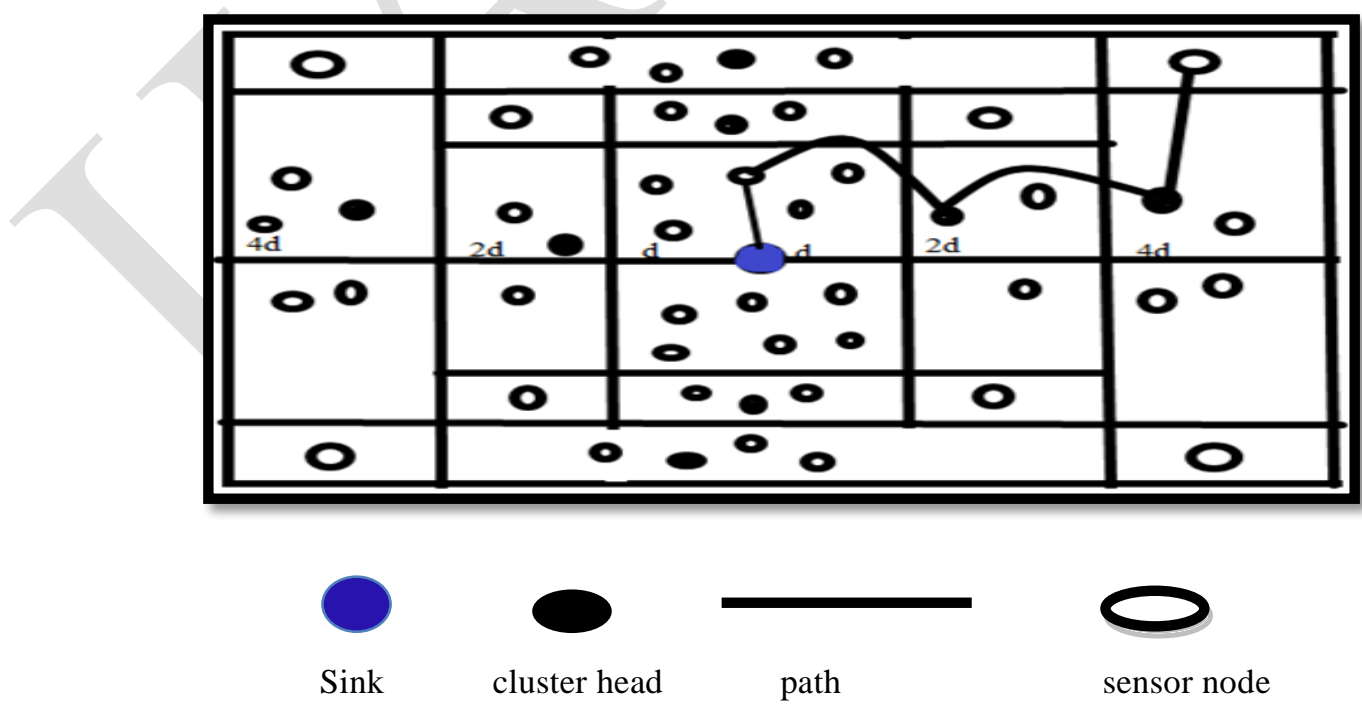

Fig.2 Existed model 
 Electronics and Instrumentation Engineering \\ (An ISO 3297: 2007 Certified Organization)}

\section{Vol. 3, Issue 8, August 2014}

\section{Routing using Relay Nodes:}

- Os will not contain relay nodes, sensor nodes of region Os will forward data to their $\mathrm{CH}$ which will aggregate data and forward it to relay node of Region Ms which then further forward data to relay nodes of Is region which then further forward data to the Base Station.

- Sensor nodes of Ms will forward data to their $\mathrm{CH}$ which then will forward data to relay node of Is region which then further forward data to base station.

- Is region will contain greater number of relay nodes as compared to Ms region. These relay nodes will receive data from the relay nodes present in middle square region and also from Cluster Head present in the middle square region and then forward it to base station.

- Therefore, the communication between Cluster Head and Relay node and between relay nodes and relay nodes takes place

- The choice to which relay node the $\mathrm{CH}$ of Is or the relay node of $\mathrm{Ms}$ or the $\mathrm{CH}$ of Ms node sends data depends upon the shortest communication distance.

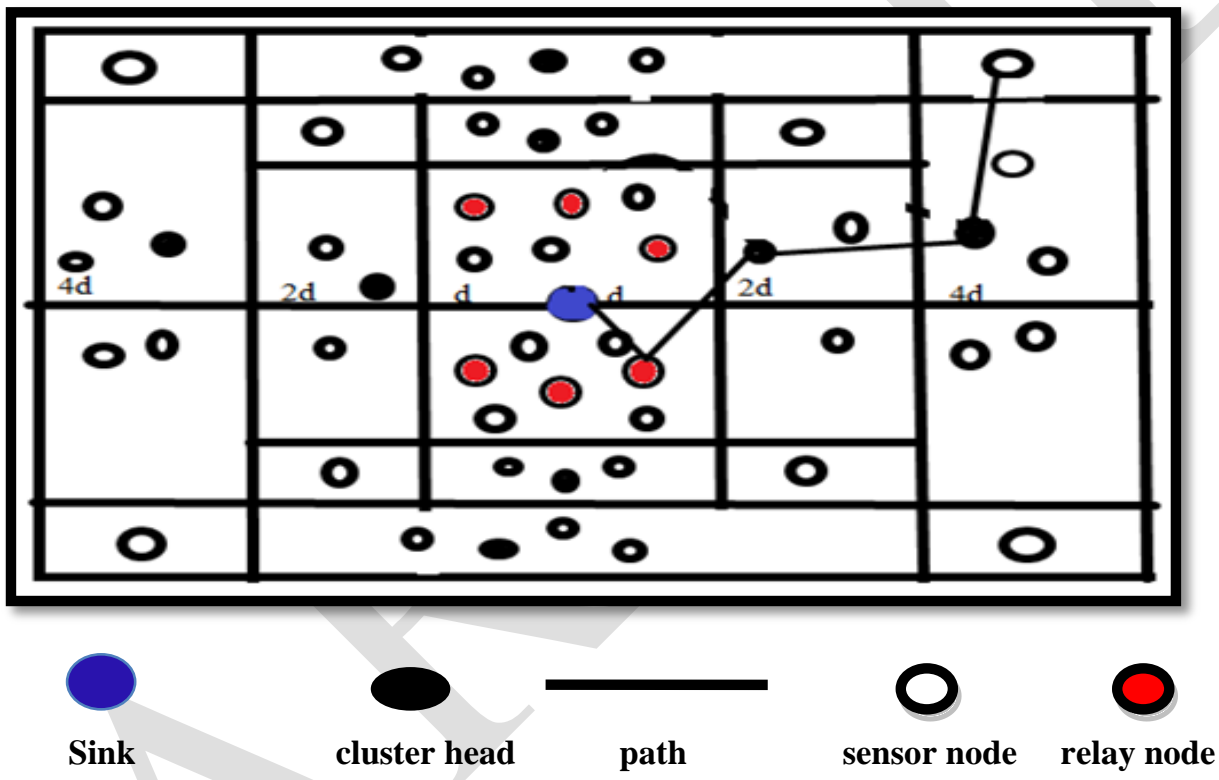

Fig. 3 Proposed Model with Relay nodes

\section{IV.SIMULATION ANALYSIS}

The Network Simulator-2 (NS-2) is an open-source simulation tool running on Unix-like operating system. It is a discrete event simulator targeted at networking research and provides substantial support for simulation of routing, multicast protocols and IP protocols, such as UDP, TCP, RTP and SRM over wired, wireless and satellite networks. The network simulator (NS), which is a discrete event simulator for networks, is a simulated program developed by VINT (Virtual Internetwork Test-bed) project group. It supports simulations of TCP and UDP, some of MAC layer protocols, various routing and multicast protocols over both wired and wireless network etc. The simulation is the technique which shows us the behaviour of modal when actually embedded into the network. Simulation of wired and wireless network functions and protocols like TCP, UDP and routing algorithms, can be done using ns2[11] 
 \\ International Journal of Advanced Research in Electrical, Electronics and Instrumentation Engineering \\ (An ISO 3297: 2007 Certified Organization) \\ Vol. 3, Issue 8, August 2014}

\section{Simulation parameters}

Table 1. Simulation parameters

\begin{tabular}{|l|l|l|}
\hline No. Item & No. Item description parameter & No. Item Description value \\
\hline 1 & Simulation Area & $800 \mathrm{X} 800$ \\
\hline 2 & No. of nodes & 41 \\
\hline 3 & No. of relay nodes & 9 \\
\hline 4 & Channel Type & Channel/wireless channel \\
\hline 5 & Antennae model & Antenna/omniantenna \\
\hline 6 & Interface queue Type & Queue/Drop tail/ priqueue \\
\hline 7 & Link layer type & LL \\
\hline 8 & Max. packet in ifq & 50 \\
\hline 9 & Initial energy of a sensor node & 80 joule \\
\hline 10 & Energy consumption & 120 joule \\
\hline 11 & Packet size & $1000 \mathrm{bytes}$ \\
\hline 12 & Simulation time & $9.0 \mathrm{sec}$. \\
\hline 13 & Transmission energy & 5 joule \\
\hline 14 & Receiving energy & 4 joule \\
\hline
\end{tabular}

\section{SIMULATION RESULTS}

The Results of the simulation are shown below. The fig. 4 shows the Simulation time vs Network Lifetime in the sensor network and the fig. 5 shows the Simulation time vs Throughput of receiving packets.

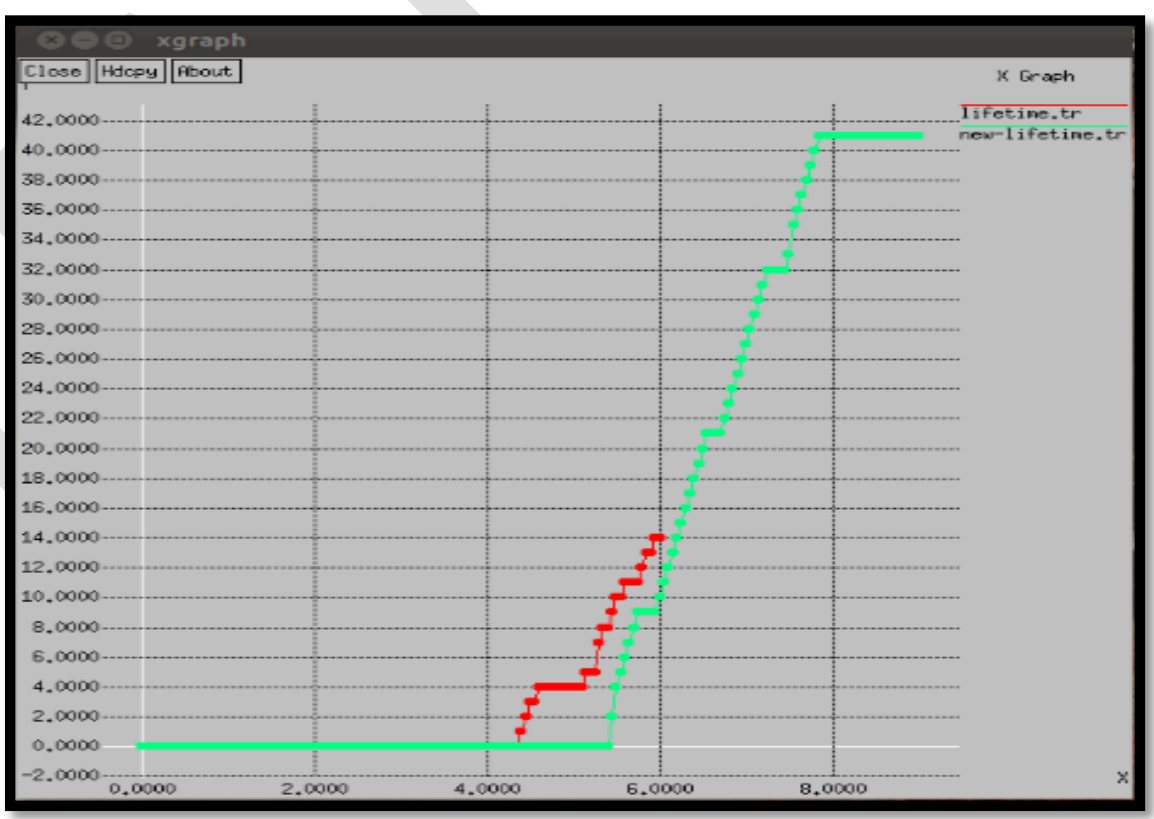

Fig. 4 Simulation time vs Network Lifetime 

Electronics and Instrumentation Engineering

\section{(An ISO 3297: 2007 Certified Organization)}

\section{Vol. 3, Issue 8, August 2014}

Above figure shows that existing technique has less network lifetime as compare to new proposed technique. Here red line shows less network lifetime of existing technique and green line shows more network lifetime. This concluded that proposed technique is better than existing technique.

The following fig. shows the simulation results of the throughput.

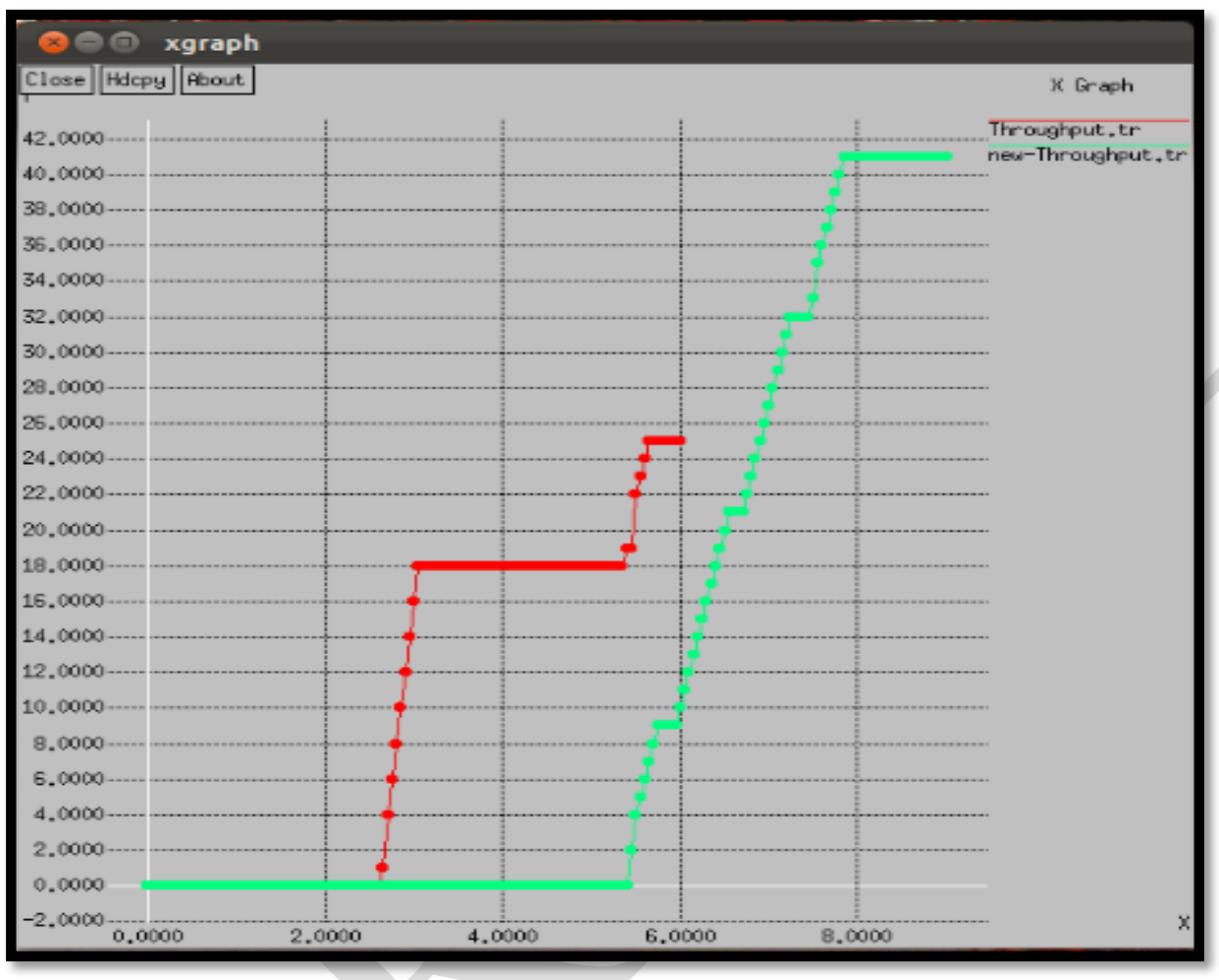

Fig.5 Simulation time vs Throughput of receiving packets

Above figure shows that existing technique has fewer throughput as compare to new proposed technique. Here red line shows fewer throughput of existing technique and green lines shows more throughput. This concluded that proposed technique is better than existing technique.

\section{VI.CONCLUSION}

The main objective of this research paper is to discuss various challenges and technique of WSN. We focused on divide and conquer technique and purposed a new novel technique. We believe that proposed algorithms discussed in this paper will give benefit for various research scholars. Its experimental results show that proposed technique gives better result which has better Network lifetime and better throughput compare to existing techniques. The results demonstrate a significant improvement in the functional lifetime of the sensor network.

\section{REFERENCES}

[1] Baydere, S., Safkan, Y., Durmaz, O. "Lifetime analysis of reliable wireless sensor networks." IEICE Trans. Comm. E88-B, 6, pp 2465-2472, 2005.

[2] Dahlila P. Dahnil, Yaswant P. Singh, Chin Kuan Ho "Energy-Efficient Cluster Formation in Heterogeneous Wireless Sensor Networks: A Comparative Study" ICACT, Feb. 2011.

[3] I.F. Akyildiz, W. Su, Y. Sankarasubramaniam, E. Cayirci. "Wireless Sensor Networks: A survey" Broadband and Wireless Networking Laboratory, School of Electrical and Computer Engineering, Georgia institute of Technology, Atlanta, GA 30332, USA Received 12 December 2001; accepted 20 December 2001. 


\section{(1) \\ International Journal of Advanced Research in Electrical, Electronics and Instrumentation Engineering \\ (An ISO 3297: 2007 Certified Organization) \\ Vol. 3, Issue 8, August 2014}

[4] Peyman Neamatollahi, Hoda Taheri, Mahmoud Naghibzadeh "A Hybrid Clustering Approach for Prolonging Lifetime in Wireless Sensor Networks" International Symposium on computer networks and distributed systems (CNDS), pp. 170 - 174, 2011 IEEE.

[5] Jakob Salzmann, Ralf Behnke, Dirk Timmermann "Hex-MASCLE - Hexagon based Clustering with Self Healing Abilities" Wireless Communications and Networking Conference (WCNC), pp. 528-533, 2011 IEEE.

[6] Ewa Hansen, Jonas Neander, Mikael Nolin and Mats Bjorkman "Efficient Cluster Formation for Sensor Networks" .pp 8-13, March 2005 IEEE

[7] K. Latif, A.Ahmad, N.Javaid, Z.A. Khan, N. Alrajeh, "Divide-and-Rule Scheme for Energy Efficient Routing in Wireless Sensor Networks" $4^{\text {th }}$ International Conference on Ambient Systems, Networks and Technologies (ANT), 2013.

[8] Vinay Kumar, Sanjeev Jain and Sudarshan Tiwari, "Energy Efficient Clustering Algorithms in Wireless Sensor Networks: A Survey", IJCSI International Journal of Computer Science Issues, volume 8, Issue 5, No 2, pp. 1694-0814, Sep. 2011.

[9] B. Mamalis, D. Gavalas, C. Konstantopoulos and G. Pantziou , "Clustering in Wireless Sensor Networks",2007 Zhang/RFID and Sensor Networks AU7777 C012.

[10] S. Tyagi, N. Kumar, "A systematic review on clustering and routing techniques based upon LEACH protocol for wireless sensor networks" Journal of Network and Computer Applications, Volume 36, Issue 2, March 2013.

[11] Robert Akl, Uttara Sawant "Grid-based co-ordinated routing in wireless sensor networks." Consumer communication and networking conference (CCNC) pp. 860-864 2007 IEEE. 